\title{
Arnold Stadler und die Reise nach Feuerland
}

In seiner spannenden Analyse der Veränderungen von Raum und Raumvorstellung am Ausgang des 20. Jahrhunderts schrieb der in Basel lebende Publizist und Kulturphilosoph Aurel Schmidt 1998:

Es beginnt uns zu dämmern, daß wir in der Falle der Immobilität und Ausweglosigkeit sitzen. Natürlich können wir nach wie vor nach Potsdam oder Palermo reisen oder nach Patagonien, das ist weiter weg, also fremder, daher verlockender, aber das ändert nichts an der Tatsache, daß jede Bewegung, die wir in einem fixierten Raum ausführen, nirgends hinführt. Die Summe ist jedes Mal die gleiche. Es ist wie in einem geschlossenen System, in dem die Energie rechnerisch weder ab- noch zunimmt, oder wie am Nordpol, wo jede Richtung nach Süden weist. ${ }^{1}$

Jede Zeit bringt ihre je eigenen Paradoxa hervor. Ungewiss in unserer Zeit des Übergangs, des Endes der vierten Phase beschleunigter Globalisierung, einer Zeit von Protektionismus und gesteigertem Nationalismus, von Erträumen ethnisch homogener Territorien und der Abschottung gegenüber all dem, was Angst vor Veränderung macht, ist freilich, auf welche Doxa das Paradoxe noch Geltung beanspruchen will und darf. Das Paradox unserer Zeit könnte jedoch darin bestehen, dass die stetig wachsende, vielleicht übergroße Beschleunigung den Eindruck der Immobilität, der Bewegungslosigkeit erzeugt, den Planwagen jener klassischen Western ähnlich, bei denen die Räder just dann stehenzubleiben oder sich gar rückwärts zu drehen scheinen, wenn diese ihre höchste Geschwindigkeit erreichen.

Ist dieser stroboskopische Effekt aber nicht ein Charakteristikum der Moderne überhaupt? Denn zeitgleich mit der historischen und räumlichen Entfaltung des Projekts der europäischen Moderne seit dem letzten Drittel des 18. Jahrhunderts hatte sich bereits der Eindruck einer Nach-Geschichte, einer Posthistoire herausgebildet, der in regelmäßigen Abständen an die Pforten des europäischen Weltbewusstseins klopft. Ihn reflektierte Georg Forster, der James Cook auf dessen zweiter Reise um die Welt begleitet hatte und etwas von den weltweiten Konsequenzen der Expansion Europas verstand, in seinem Beitrag 'Die Nordwestküste von Amerika und der dortige Pelzhandel', in dem er auf die Folgen des europäischen (Welt-) Handels aufmerksam machte, „in welchen sich allmählig die ganze Weltgeschichte aufzulösen“ scheine. ${ }^{2}$

1 Schmidt, Aurel: Von Raum zu Raum: Versuch über das Reisen. Leipzig: Merve 1998, S. 38.

2 Forster, Georg: Die Nordwestküste von Amerika, und der dortige Pelzhandel. In (ders.): Werke in vier Bänden. Herausgegeben von Gerhard Steiner. Bd. 2: Kleine Schriften zur Naturgeschichte, Länder- und Völkerkunde. Ansichten vom Niederrhein. Leipzig: Insel Verlag 1971, S. 258; vgl. hierzu

Ә Open Access. (C) 2020 Ottmar Ette, publiziert von De Gruyter. (cc) BY-NC-ND Dieses Werk ist lizenziert unter der Creative Commons Attribution-NonCommericial-NoDerivatives 4.0 Lizenz.

https://doi.org/10.1515/9783110650686-030 


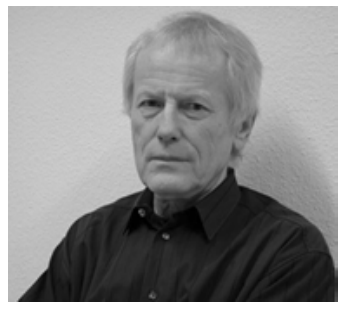

Abb. 93: Aurel Schmidt (1935, Berlin).

Auch der französische Historiker Jules Michelet bewegte sich ein gutes halbes Jahrhundert später in einer Zeit der posthistoire, die sich schon nach den großen Revolutionen wähnte. Und doch dreht sich unsere Geschichte, dreht sich unser Planet mit einer irrwitzigen Geschwindigkeit, die wir anhand des Klimawandels oder des Artensterbens messen und protokollieren, aber nach bisherigen politischen Parametern nicht zu stoppen vermögen. es sei denn, ein guter Teil der Menschheit gelangte zu der Einsicht, dass wir im Zeichen des Wachstums nicht länger wachsen dürfen. Die rasende Geschwindigkeit, der wir allenthalben ausgesetzt sind, aber wird man nur zum eigenen Schaden sowie zu dem unseres Planeten leugnen dürfen. Doch Parteien gibt es freilich hierfür auch, in Hülle und Fülle sogar.

Die Erfahrung nach-geschichtlicher Zeiten scheint auf eine besondere, bislang wenig ergründete Weise mit der Erfahrung geschichtlichen Denkens verbunden zu sein. Höchste Beschleunigung und Empfindung des Stillstands - dies zeigen auch die Ereignisse beim Fall der Berliner Mauer sowie die drei nachfolgenden Jahrzehnte grundlegender rapider Veränderungen in den als 'neu' bezeichneten Bundesländern besonders eindrücklich - sind keinesfalls voneinander getrennte oder gar einander diametral gegenüberstehende Phänomene unserer Geschichte. Die Verschränkung von Akzeleration und Ruhe bildet ein Paradoxon, das gewiss zur Grunderfahrung der Moderne zählt und sich nicht zuletzt in der so unterschiedlich modellierten Figur des Reisenden manifestiert. Ja, man könnte mit guten Gründen sogar sagen, dass sich in der Figur oder den Figuren von Reisenden wie in einem Brennpunkt die unterschiedlichsten Isotopien unseres geschichtlichen Erlebens schneiden und überkreuzen.

Angesichts dieser Überlegungen des französischen Mythenkritikers und Zeichentheoretikers Roland Barthes, der die absolute Ruhe des Überschallpiloten

Lepenies, Wolf: Das Ende der Naturgeschichte. Wandel kultureller Selbstverständlichkeiten in den Wissenschaften des 18. und 19. Jahrhunderts. Frankfurt am Main: Suhrkamp 1978, S. 118. 
hervorhob, drängt sich eine Frage auf: Ist an die Stelle des jet-man der Moderne heute die Figur des chat-man der Postmoderne, der sich im WorldWideWeb mit kaum noch zu bemerkender Zeitverzögerung weltweit bewegende Nutzer des Internet getreten? Hat die Atmosphäre des Welt-Raums ihre Dominanz an die Logosphäre des chat-rooms in Echtzeit abgetreten, ohne doch für die Nutzerinnen $\mathrm{zu}$ verschwinden? Hat das Jetten als Mythos des Alltags ausgedient und dem Chatten im Cyberspace Platz gemacht? Sind wir zu Gewohnheitstieren planetarischer Ubiquität im Netz geworden, die uns über die tatsächliche Präsenz auf unserem Planeten sanft hinwegtäuscht? Oder anders: Glauben wir uns weltweit vernetzt - und sind doch nur mit einem kleinen Teil der Erdbevölkerung wirklich in Kontakt?

Selbst Superman, Batman und Spiderman als vermeintlich unverwüstliche Mythen der Geschwindigkeit und der Überwindung des empirischen Raums scheinen in der Tat ihre Vorherrschaft dem neuen künstlichen Mythos vom Menschen im virtuellen Netz überlassen zu haben. Gerade das Beispiel weltweiter kommunikativer Vernetzung mag zeigen, dass auch die verstärkte Vielbezüglichkeit unterschiedlichster Räume trotz der immer höheren Kommunikationsgeschwindigkeit mit der Erfahrung und Wahrnehmung von Bewegungslosigkeit, von Stillstand, von einem 'Durchdrehen' der Räder und nicht zuletzt von einem Verschwinden des Raumes verknüpft sein kann. Die Kommunikationsgeschwindigkeiten in der weltweiten Zirkulation von Ideen faszinieren und täuschen zugleich darüber hinweg, wie verengt die Erfahrungs- und Erlebenswelt unseres weltweiten Gewebe heute ist und uns über konkrete raumzeitliche Erfahrungen und Erlebnisse hinweglügt. Macht ein in einem konventionellen Sinn verstandenes Reisen dann überhaupt noch einen Sinn?

Vom Raum der Gedanken, vom virtuellen Raum zirkulierender Vorstellungen her betrachtet - also gleichsam nicht von der Hardware, sondern von der Software her gesehen -, ist der tour du globe beziehungsweise der tour de l'univers (im Sinne von Denis Diderot) gerade nicht zu einem Stillstand gekommen. Die Beschleunigung in der Empirizität der Atmosphäre wird von der Virtualität weltweiter Kommunikation in der Logosphäre beständig in Bewegungslosigkeit umgedeutet, eine Erfahrung, die mit der europäischen Moderne heraufzieht, welche sich ihrerseits erst vor dem Hintergrund von Welt-Erfahrung konstituieren kann. Macht in diesem Zusammenhang also ein konkretes Reisen in Raum und Zeit noch Sinn? Ist Reisen also noch für uns zeit-gemäß, gerade auch vor dem Hintergrund immer stärker veränderter Klimadaten, die schon zum Phänomen der 'Flugscham' geführt haben?

Wie Aurel Schmidt am Beispiel Goethes aufzeigte, „wird das Reisen zu einer Technik und Methode der unausweichlichen Selbstverwandlung und 
Selbsterneuerung“3 - doch ist damit im Sinne Diderots sicherlich nicht die einzig mögliche Technik und Methode bestimmt. Schmidts Überlegungen am möglichen Ende jenes Raumes der europäischen Moderne, an deren Anfang sich Texte wie Diderots Supplément au Voyage de Bougainville und mehr noch Jacques le fataliste et son maître ansiedelten, fällt am Jahrtausendende weniger selbstgewiss und zukunftsfroh aus als jene der beiden Dialogpartner des 18. Jahrhunderts: Der Mensch reist nicht, er „wird transportiert, abtransportiert, deportiert“, verbleibt „stets am gleichen Ort, in der Leere und Langeweile, in die er verstrickt ist“. ${ }^{4}$ Wird damit letztlich nicht das Reisen als eine wichtige Kulturtechnik des Menschen obsolet, der sich mit anderen, verschiedenartigen Kulturen beschäftigen und auseinandersetzen möchte?

Auch hier scheint, allerdings vom anderen Ende dieses Raumes her, die Grenze zwischen jenen, die aufbrechen, und jenen anderen, die zu Hause bleiben, nicht nur brüchig, sondern letztlich irrelevant geworden zu sein. Aber ist es wirklich gleichgültig, ja sogar gleich gültig, ob wir uns zu den Reisenden oder den Daheimgebliebenen - eine Scheidung, die unsere gesamte Vorlesung durchzog - zählen dürfen?

Gleichwohl ist diese Art der Reise im Gegensatz zur überwiegenden Mehrzahl von Migrationsbewegungen in umgekehrter, von den 'Peripherien' in die 'Zentren' verlaufender Richtung eine selbstgewählte, von ihrer Etymologie her als sportlich zu bezeichnende De-Portation: Der Weg vom lateinischen portare zum deutschen Sport führte - wie der Blick in ein etymologisches Wörterbuch zeigt - über das mittellateinische deportare, das mittelfranzösische desporter sowie das neuenglische sport. ${ }^{5}$ Gerade diese sportlichen Fernreisen beinhalten überdies eine zumeist unbewusste Zoologisierung des Anderen. Denn wie im Zoo wird das Unbekannte, das Exotische, das Fremdartige besucht und besichtigt, nicht aber zum Gegenbesuch außerhalb der Umfriedung der Zoogitter eingeladen. Ein spätes Erbe unserer sogenannten 'Völkerschauen'? Touristen bleiben nach ihren Ausflügen wie die Rückkehrer aus dem Zoo gerne unter sich. Nicht überall sind auf unserem Planeten die Bewegungsmöglichkeiten und die Lebenschancen miteinander vergleichbar - und auch dies ist von Beginn an ein Bestandteil jenes Paradoxons, das die europäische Moderne in ihrer beständigen Zunahme an Raumumfang und Reisegeschwindigkeit gebildet hat. In leichter Veränderung einer wohlbekannten Formulierung ließe sich folglich sagen: Das Empire reist zurück.

3 Schmidt, Aurel: Von Raum zu Raum, S. 23.

4 Ebda.

5 Vgl. Kluge: Etymologisches Wörterbuch der deutschen Sprache. 23., erweiterte Auflage. Bearbeitet von Elmar Seebold. Berlin - New York: Walter de Gruyter 1999, S. 782. 
Die Ausführungen Aurel Schmidts bringen im Eingangszitat scheinbar zufällig Patagonien, jene Region, die Arnold Stadlers Feuerland zu umschreiben versucht, in einen wohl rein alphabetisch begründeten Zusammenhang mit Palermo und überdies mit Potsdam, meinem innerhalb dieses Dreiecks keineswegs bedeutungslosen Ort des Lesens. Patagonien, ${ }^{6}$ Mittelmeer und Mitteleuropa werden durch einen seltsamen Zufall in eine Beziehung zueinander und zugleich mit der Problematik der Reise gesetzt, eine Relation, die sich auch in dem 1992 erschienenen Roman des unweit des badischen Meßkirch geborenen süddeutschen Autors entdecken lässt. Mit anderen Worten: Die von Aurel Schmidt skizzierte Raumproblematik scheint geradezu gemünzt auf Arnold Stadlers in siebenunddreißig unterschiedlich kurze und römisch durchnummerierte Kapitel (oder Stationen) unterteilten Kurzroman. Dieser soll nun auf den nachfolgenden Seiten einer näheren Untersuchung unterzogen werden, um aus ihm Lehren für die künftige Entwicklung von Reiseliteratur zu ziehen.

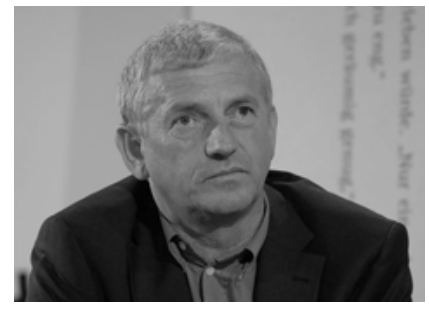

Abb. 94: Arnold Stadler (Meßkirch, 1954).

Arnold Stadlers Roman Feuerland bildet den zweiten Teil einer autobiographischen Trilogie, die 1989 mit Ich war einmal begann und 1994 mit Mein Hund, meine Sau, mein Leben abgeschlossen wurde. ${ }^{7}$ Es handelt sich um einen Text, der von Reisebewegungen jeglicher Art charakterisiert und gequert wird, wobei Thema, Struktur und Strukturierung der Reise topisch ebenso mit der Problematik der Lebensreise als Sinnsuche wie mit jener des Todes als Endpunkt jeglicher irdischen Reise verknüpft werden. Reisen erzeugen Leben ebenso wie sie

\footnotetext{
6 Vgl. zu Literatur und Reiseliteratur rund um Patagonien Haase, Jenny: Patagoniens verflochtene Erzählwelten. Der argentinische und chilenische Süden in Reiseliteratur und historischem Roman (1977-1999). Tübingen: Max Niemeyer Verlag 2009.

7 Einen ersten Überblick über das Werk des 1954 geborenen Autors bietet Knittel, Anton Philipp: Arnold Stadler. In: Kritisches Lexikon zur deutschsprachigen Gegenwartsliteratur. 53. Nachlieferung. München: Edition text + kritik 1996.
} 
den Tod auslösen: Leben heißt in diesem Sinne ein unverändertes Reisen, gleichviel, ob dieses in seiner Vektorizität gerichtet oder ungerichtet ist.

Diese Vektorisierung aller Lebensbezüge zeigt sich bereits in den fulminanten Anfangssätzen dieses trotz seines geringen Seitenumfangs beunruhigend sperrigen, eine ganz eigene sprachliche Welt erzeugenden Erzähltextes. Der Anfang markiert ein Lebensende, wie man es sich stärker von Bewegungen durchzogen kaum vorstellen könnte:

In der Nacht vom 20. zum 21. Juni warf sich der Sohn des Fellhändlers Antonio aus Pico Grande, Patagonien, vor den Zug. Es war sein erstes Lebenszeichen.

Trotz der Verspätung des Nachtzuges von Esquel nach Bahia Blanca wartete der Kandidat im Chevrolet seines Vaters, den er sich für diesen Zweck geliehen hatte, bis er den Zug kommen hörte. Dann schlug er die Tür des Lieferwagens zu, warf die Fahrzeugschlüssel zusammen mit seinem ganzen Schlüsselbund in die Pampa, rannte die wenigen Meter bis zu den Schienen und legte sich gegen die Fahrtrichtung, aber parallel zu den Gleisen, mitten auf den Boden. Es war eine Sache von Sekunden, und er hatte alles überstanden. Diese Bahnlinie war die einzige Verbindung der Gegend mit der Welt. ${ }^{8}$

Solch ein Romanauftakt wirkt, verbunden mit der lakonischen Erzählweise, wie ein Paukenschlag. Die Leserschaft wird mit wenigen Worten eingefangen und gefesselt - oder vielleicht auch abgestoßen. Bereits in diesen Anfangssätzen, in diesem incipit, sehen Sie es schon: Speed kills. Charakteristisch ist in diesem ausgefeilten Beginn nicht nur die präzise Einführung der Themen des Selbstmordes und des Todes, die als basso continuo den gesamten Text begleiten, sondern die Verknüpfung dieser Thematik mit verschiedenen Verkehrs- und Transportmitteln und dadurch der Problematik der Raumveränderung insgesamt. Der Mensch erscheint als Teil einer Welt, die von genau getakteten Bewegungen durchzogen wird: Man kann sich auf sie, leichte Verspätungen einkalkulierend, verlassen.

Vektorizität ist niemals unschuldig: Bewegung erscheint von Beginn an als eine Bewegung zum Tode, die paradoxerweise zum ersten Lebenszeichen der ersten Romanfigur gerät. Auch hier sind, gewiss auf andere Weise, höchste Beschleunigung und Bewegungslosigkeit unmittelbar zusammengedacht. Im Selbstmord prallen beide Bewegungsformen aufeinander. Die einzige Verbindung der Region mit der Welt auf einer zumindest analogen Ebene führt zum Abbruch der Verbindungen des Individuums mit der Welt, auch wenn dies aus zugtechnischen Gründen verspätet erfolgt. Die Verbindungen werden abgebrochen, selbst die Schlüssel des Automobils werden weggeworfen, als sollte niemand mehr dieses

8 Stadler, Arnold: Feuerland. Roman. Salzburg: Residenz 1992, S. 7. 
Fahrzeug für andere Reisen und Bewegungen mobilisieren können. Natürlich wird dies dessen erneute Inbetriebnahme höchstens verzögern.

Nicht weniger paradox ist, dass der endgültige Stillstand, der auch zu einer Zerstückelung des Körpers führt, erst durch eine Reihe mehr oder minder abrupt beschleunigter Bewegungen erzeugt wird. Wir wohnen einer Versuchsanordnung mit todsicherem Ausgang bei: Der Zug wird als Emblem des Fortschritts und der Moderne zum Henker eines Lebens, das sich aus seinen ungeordneten Bewegungen anders nicht zu retten weiß.

Für die sich bereits in den Anfangssätzen abzeichnende Konstellation ist nicht unwesentlich, dass nicht nur in der Eingangsszene, sondern auch am Ende des Romans die verschiedenen Todesarten in jenem Teil des Planeten, der vom Erzähler immer wieder als das 'Ende der Welt' bezeichnet wird, sich mit Vorliebe an jenen Leitlinien der Infrastruktur in Szene setzen oder ereignen, welche „die einzige Verbindung der Gegend mit der Welt“ darstellen. Mit einer wohlkalkulierten Schärfe werden die unterschiedlichsten Reisebewegungen und Vektorizitäten der handelnden Figuren immer wieder glatt in ihren jeweiligen Mobilisierungen durchtrennt. Begeht gleich zu Beginn des Romans der Sohn des 1938 aus Deutschland nach Argentinien emigrierten Onkels des Ich-Erzählers Selbstmord, so wird ein anderer Auswanderer, in eben jenem Augenblick, als er seinen Wohnort Pico Grande in Patagonien verlässt, um nach Deutschland zurückzukehren, während einer Autofahrt im Schlaf vom Tod ereilt. ${ }^{9}$ Die Bewegungsfiguren vererben sich gleichsam transgenerationell: was in unserer Familie vektoriell angelegt und damit gebahnt ist, kann sich auf gebahnten Wegen einschließlich der Eisenbahn jederzeit in unser Leben fressen und es bestimmen. Vektoren sind transgenerationelle Bahnungen.

Die Bahnungen in Arnold Stadlers Feuerland sind minuziös geplant. In einer tödlichen Symmetrie und Choreographie von Romananfang und Romanende ist die Bewegung stets eine Bewegung zum Tode. Ganz bewusst führen von der Eingangs- und Schlussszene direkte Beziehungen zum Ich-Erzähler, der gleich zu Beginn des letzten Kapitels festhält: „so könnte auch meine Geschichte enden. “10 Mithin weist der Roman eine mehr oder minder verdeckte serielle und relationale Struktur auf, die - wie noch gezeigt werden soll - genealogisch und transgenerationell fundiert wird. Für unverrückbare Identitäten bleibt in diesem Seriellen Spiel kaum mehr als ein transitorischer Raum. In den Figuren des Romans sind Bahnungen angelegt, denen diese Figuren nicht auszuweichen vermögen.

9 Ebda., S. $153 \mathrm{f}$.

10 Ebda., S. 153. 
Doch die hermeneutische Bewegungsfigur, die Kon-Figuration der Bewegungen des Ich-Erzählers sticht gleichwohl scharf von den Bewegungsmustern aller anderen Figuren des Romans ab. Denn die Grundfigur seiner Bewegungen ist ganz einfach der Kreis. Er bricht von Deutschland, vom badisch-schwäbischen Alpenvorland aus auf, reist nach Argentinien, wo er sich einen Südhalbkugelsommer lang im patagonischen Andenvorland aufhält, und kehrt schließlich wieder in seine 'Heimat' nach Deutschland zurück. Und an diese Figur sind gleichsam die Elemente und Aspekte des reiseliterarischen Berichts gekoppelt.

Kein Zweifel: Wir haben es hier mit einer Globalisierung auf touristischer Ebene zu tun. Dies ist gleichsam eine transversale Globalisierung, die zwischen Globalisierung von oben und Globalisierung von unten steht, wobei sie sich freilich der ersteren näher weiß. Denn der Ich-Erzähler unternimmt seine Reise auf eigenen Wunsch, nach eigenen Vorstellungen - und dies ist bei der Mehrzahl der auf unserem Planeten getätigten Reisen keineswegs der Fall. Nein, hier geht es nicht um diskontinuierliche Sprünge, wir haben es vielmehr mit einer zielgerichteten kontinuierlichen Bewegungsfigur zu tun.

Arnold Stadlers Text vollzieht damit jene 'Ur-Bewegung' des europäischen 'Besuchers' in Amerika nach, die seit Cristóbal Colón die eigene Reise im Zeichen der Rückkehr und damit im Zeichen des Kreises begreift. Es ist die europäische Bewegungsfigur par excellence. Nicht von ungefähr erinnert die Kapitelstruktur mit ihren im Inhaltsverzeichnis aufgelisteten narrativ gehaltenen Überschriften, die von 'Wie der Sohn des Fellhändlers starb' bis 'Wie die Geschichte endet' reichen, ebenso an den Schelmenroman, der dem Reisebericht unendlich viel schuldet, wie an den Reisebericht selbst, dessen Bewegungen die Kapitelüberschriften nachvollziehbar machen. Erneut erweisen sich die novela picaresca, aber auch autobiographische Schreibformen als Korrespondenzformen reiseliterarischen Schreibens, in denen wir auf Grundelemente des Movens von europäischer Reiseliteratur stoßen.

Das intertextuelle Netzwerk des reiseliterarischen Romans ist fein gesponnen und spielt eine Vielzahl von gerade auch reiseliterarischen Bezügen ein. Nicht nur die Anspielungen auf das Menschenfressertum, die den Erzähler seit seiner Kindheit quälen, sondern auch die durch die Überschriften gegebene Präsentationsform stellen eine direkte Verbindung zu Hans Stadens berühmtem und in unserer Vorlesung ausführlich behandelten Bericht aus dem 'Brasilien' der Mitte des 16. Jahrhunderts her, in welchem sich viele Kapitelüberschriften dieses Typs ausmachen lassen. Was auf Brasilien gemünzt war, hätte auch für Patagonien oder Feuerland gelten können: 'Wie wir herausfanden, in welchem Teil des fremden Landes wir Schiffbruch erlitten hatten'. Stadens wie Stadlers Texte sind Schiffbrüche mit Rückkehr, wenn auch nicht für alle der Reisenden an Bord. Die Reisen der ersten Phase beschleunigter Globalisierung sind in den 
Reisen der vierten Phase unmittelbar präsent und bilden einen sinngebenden Horizont, in dessen Rahmen sich das jeweils Erzählte einfügt.

Doch vergessen wir nicht, dass die Reisen unserer Tage zugleich auch den Bahnungen früherer Zeiten folgen und eine Reise damit stets auch transgenerationell motiviert ist. Am Anfang der Reise des Ich-Erzählers stand die Reise eines anderen, des Onkels Antonio, der 1938 ,fortgefahren und nicht wiedergekommen“ war, so wie es wiederum dessen Onkel getan hatte, der 1898 - gleichsam zum Zeitpunkt der dritten Phase beschleunigter Globalisierung - den Flecken Nueva Alemania, das später in Pico Grande umbenannt wurde, gegründet hatte. Verschiedene Zeitebenen überlagern sich, werden wie in einem $\mathrm{Pa}$ limpsest gleichzeitig lesbar und beleuchten sich wechselseitig: un voyage peut en cacher un autre.

Im Falle des Ich-Erzählers sind die transgenerationellen Bahnungen ganz konkreter Art. Durch die Briefe von Onkel Antonio wurde das neudeutsche Amerika für den Ich-Erzähler von Kindesbeinen an zum Zielpunkt eines anhaltenden Fernwehs. Der Onkel kam nicht mehr zurück; dafür schickte er Briefe, die die Phantasie des Jungen beflügelten. Damit trat die Schrift an die Stelle der räumlichen Bewegung, ein Schreiben also, das virtueller Ersatz für die Bewegung und zugleich deren Folge und Ausdruck ist.

Und doch hatten diese Briefe auf den ersten Blick wenig Verheißungsvolles, wenig, was das Fernweh nach einem ganz anderen Land hätte wecken können, ist doch „im Grunde alles ganz wie zu Hause“. ${ }^{11}$ Die Ent-Täuschung durch eine Reise scheint vorprogrammiert. Allerdings sollte uns diese ungezählte Male wiederkehrende Formel nicht über deren ambivalente Form hinwegtäuschen, insoweit sich doch eine grundlegende Differenz einschleicht, welche aus dem wie zu Hause zugleich den Gegen-Raum zum Zuhause macht. Denn im Gleichen, das nicht das Selbe ist, gewinnt das Eigene den Charakter eines Anderen, das die Schafe nicht zu Kühen, die Anden nicht zu Alpen, das Neudeutsche nicht zum Deutschen werden lässt. Eine Differenzqualität schiebt sich dazwischen, welche gerade am wie zuhause ansetzt und durch ein aber doch ganz anders vervollständigt.

Die Überwindung des realen, des empirischen Raumes kann die in sie gesetzten Hoffnungen der Grenzüberschreitung zum ganz Anderen gleichwohl nicht erfüllen. Der Ort der Ankunft ist Ent-Täuschung. ${ }^{12}$ Schon „mit Beginn der Reise“ war das Reisefieber verflogen, ${ }^{13}$ aufgebraucht. Die autobiographischen,

\footnotetext{
11 Ebda., S. 11.

12 Ebda., S. 13.

13 Ebda., S. 17.
} 
in die Zeit der Vergangenheit weisenden Spuren überlagern immer wieder die reiseliterarischen, am Raum der 'Neuen Welt' sich ausrichtenden Bewegungen mit ihrer eigenen Vektorizität. Die Transarealität dieser transatlantischen Bezüge zwischen Alpenvorland und Andenvorland ist evident.

So war die Neue Welt zwar in der Alten Welt dem Kind als eine neue erschienen; doch wird sie dem Erwachsenen just in der Neuen Welt zur Alten. Eine Reise im eigentlichen, traditionellen Sinne ist beim Protagonisten als bewusster Prozess kaum wahrnehmbar; denn selbst bei der Überquerung des Atlantik, den Onkel und Uronkel noch auf Auswandererschiffen in wochenlangen Reisebewegungen überwunden hatten, ist der Ich-Erzähler schlicht eingeschlafen und nimmt die Bewegung im Raum aus einer Höhe von zehn Kilometern kaum war. Die Bewegung des Flugzeugs gerinnt bestenfalls zum fixierten philosophischen Topos, zum „Aussichtspunkt wie über dem Meer“, auf dem der Reisende sitzt und über die Bewegungen anderer, seiner Vorfahren, nachsinnt. ${ }^{14}$ Fast will es scheinen, als wäre der Reisende der eigentliche Daheimgebliebene, als wäre das Diderot'sche Paradox des Reisenden von neuem wirksam. Die Verbindung von Reisen und Schreiben scheint gestört, vielleicht sogar zerstört.

Anders als in einer Welt, deren Konturen und Umrisse durch die Entdeckungsfahrten des ausgehenden 18. Jahrhunderts noch nicht gesichert waren, ist die Utopie am Ende der Welt nun an ihr Ende gelangt. Es scheint, als ob sich am vermeintlichen Ende der Welt, das seinerseits ein anderes Ende noch weiter nach Süden projiziert, die Welt für den Protagonisten zu einer Kugel gerundet hätte, die kein Ende kennt und in ihrer wahrhaft erfahrenen Endlichkeit der Utopie als dem 'reinen' Anderen endgültig den Garaus machte. Gefangen in einer Welt, die sich zur Kugel rundet und aus der es kein Entrinnen gibt: Wir befinden uns am Gegenpol zu einer Utopie, nicht an einem Nicht-Ort, sondern an einem Ort, der eigentlich zwei Orte ist, im Anden- und im Alpenvorland liegt. Die Überlagerung der Räume als Folge einer willentlichen De-Portation aber ist nicht gleichbedeutend mit deren Ineins-Fallen. Distanz und Differenz sind nicht getilgt. Die Überlagerungen dieses antipodischen Feuerlands beinhalten stets ein Spiel von Identität und Differenz, an dem sich das Schreiben in einer ständigen Grenzüberschreitung zwischen beiden Polen abarbeitet. Das Reisen entzieht dem Schreiben jegliche Möglichkeit von bunten Bildern, führt an den immer gleichen Ort, an welchem sich die Landschaften überlagern und nur das Subjekt sich in diese Doppel-Landschaft zu fügen sucht.

Doch es gibt auch andere Bewegungsmuster in diesem reiseliterarischen Roman Arnold Stadlers. Denn die familiären Bahnungen stellen übereinander

14 Ebda., S. 149. 
liegende Reiseverläufe dar, die sich wie unterschiedliche Vektorizitäten, gespeicherte Bahnungen, beschreiben lassen. Die eigene Familiengeschichte des Protagonisten präsentiert sich als eine Abfolge von Auswanderungen, waren doch nicht nur Onkel und Uronkel nicht mehr an ihren Herkunftsort zurückgekehrt, sondern auch jener entfernte Vorfahre „aus der Gegend von Schwaz“, ${ }^{15}$ der irgendwann (als eine Art Anton) aus Tirol aufgetaucht war, sich nördlich der Alpen niederließ und die Familie mit seinem im Roman lange Zeit verschwiegenen Nachnamen Schwanz genealogisch und patriarchalisch zeugte und prägte. Gegen Ende des Romans zeigt sich, wie nachhaltig diese genealogische Prägung nicht allein die Namensstruktur der Familie in beiden Welten durchläuft:

Es ist an der Zeit, daß wir unseren Namen preisgeben. Für wen halten uns die Leute?

Du heißt Rosa, Rosa Schwanz heißt du, nach deinem Vater, einem Schwanz wie ich. Wir alle stammen von jenem Schwanz ab, der aus Tirol in unser Haus kam, nur ein Müllersknecht.

Das ist die Wahrheit.

Nun konnte ich Rosa verraten, daß man schon in Chile über mich gelacht hatte, als ich als Ziel meiner Reise Pico Grande nannte, wörtlich übersetzt: Groß-Spitz, metaphorisch: Groß-Schwanz, etwa so als deutsches Bild. Rosa wußte weder, daß man in Chile jenen Teil, der bei uns topographisch-metaphorisch Schwanz genannt wird, als pico bezeichnet. Noch wußte sie, daß die Schwanz-Familie, die Schwanz-Seite (die Mutter war ja eine Indianerin), auf die sie sich so stolz berief, zu Hause nur ein Gelächter eintrug. Ganz zu schweigen von ihrem vollständigen Namen. ${ }^{16}$

Genealogien überlagern weithin eine Familiengeschichte, die sich transatlantisch fortpflanzt und doch immer wieder in dasselbe Grundmuster zurückfällt. Die unilinearen Bewegungsmuster dieser Familiengeschichte verweisen auf bewusste Entscheidungen, angesichts schwieriger Lebenskontexte das Eigene zu verlassen und an einem anderen Ort ein neues Leben aufzubauen. Doch zugleich ergeben sich Strukturen, die von willentlichen Entscheidungen nicht beeinflussbar sind und im gleichen Maße die Herkünfte und Abkünfte familiär vorantreiben. Sie sind jeweils mit Reisen und dem bewussten Willen, einer Situation zu entfliehen, gekoppelt.

So war auch schon die Stammmutter Lys, die auf dem Auswandererschiff den Uronkel des Protagonisten und späteren Gründer von Nueva Alemania kennengelernt hatte, vor einer Hungersnot in der Schweiz geflohen, hatte also ihr nacktes (Über-) Leben gerettet, um sich noch einmal einen neuen Raum des Eigenen schaffen zu können. Gelang diese Flucht, gelang dieses Ausbrechen aus einer Fatalität, die Lys einst hinter sich lassen wollte? Die Erzählweise der Hauptfigur lässt kaum

15 Ebda., S. 21.

16 Ebda., S. 131. 
den Schluss zu, dass diese linearen Bewegungsmuster ohne Rückkehr zum Lebensglück geführt hätten. Vielmehr scheinen all diese Einzelgeschichten auf dem Familienfriedhof von Pico Grande zusammenzulaufen, sich auf der Nekropole des Grabhügels mit seinen schönen Grabsteinen und ihren deutschen Inschriften aufzutürmen.

Denn die Hoffnung darauf, das Gleiche unter anderem Himmel als das Neue entwickeln zu können, scheitert wie so viele andere Hoffnungen, verschwindet ebenso wie der Ortsname Nueva Alemania, der nach Ende des Ersten Weltkrieges nicht mehr opportun ist und mitsamt seinen großen Ambitionen ausgelöscht wird. Wenig, ja gar nichts bleibt vom großen Traum, sich in der Neuen Welt eine bessere Welt, ein besseres Leben aufzubauen. Nicht nur die unterschiedlichen Leben der Familienangehörigen wirken deplatziert, auch ihre Hoffnungen und Wünsche sind es.

Dies gilt auch für den Ich-Erzähler selbst; doch besitzt er im Gegensatz zu seinen Vorfahren ein Rückflugticket, das es ihm erlaubt, die Vorgänge und Geschichten in Amerika aus der Perspektive des Reisenden und des Gastes und damit aus einer zumindest materiell gesicherten Distanz zu betrachten. Mag sein, dass damit auch die bisweilen selbstironische, bisweilen sarkastische Erzählweise wie auch die Modellierung der Erzählerfigur zusammenhängt, die sich in die Erzähltrilogie einfügt, ohne ihre Eigentümlichkeit zu verlieren. Doch der Ich-Erzähler gräbt tiefer und tiefer in der Familiengeschichte.

Figuren treten aus dem Dunkel der Geschichte und werden zu handelnden Personen. Ein zuvor nur durch die Briefe des Onkels „geisternder Mensch“, ${ }^{17}$ Fritz alias Friedrich Wilhelm von Streng, nimmt durch den Besuch in Pico Grande für den Protagonisten konkrete Gestalt an. Sein gewaltsamer Tod setzt den letzten Akzent des Buches; doch selbst die wenigen Biographeme und Notizen eines Lebens, das den einzigen Sohn eines Generaldirektors nach einer zunächst standesgemäßen Laufbahn 1936 aufgrund seiner jüdischen Herkunft und seiner Homosexualität aus der Bahn warf und den nunmehr Verfolgten dazu zwang, „mit unbekanntem Ziel das Deutsche Reich“ zu verlassen, ${ }^{18}$ lassen ein anderes Muster diskontinuierlicher Reisebewegungen erkennen. Nicht willentliche und bewusste Entscheidung, sondern erzwungene Flucht bestimmt diese Art von Lebensweg, der sich am Ende mit der Entscheidung zur Rückkehr doch noch einmal abrunden will, dann aber jäh und plötzlich durch den Tod abgebrochen wird. Hier beginnt der Roman Arnold Stadlers, als Rückblick auf das 20. Jahrhundert endgültig zum Rückblick auf ein Jahrhundert der

17 Ebda., S. 33.

18 Ebda., S. 35. 
Migrationen zu werden. Feuerland präsentiert eine Geschichte von Migrationen, Flucht und Exil, von Ansiedelung, Besiedelung und Hoffnung, Ausweglosigkeit und Tod. Es ist die Geschichte des vergangenen Jahrhunderts im transatlantischen Spannungsfeld.

Doch auch für diese Geschichte gibt es eine Romanfigur, in der sich gleichsam alle Bewegungen überschneiden. Im anschließenden Kapitel stößt der Erzähler auf Galina Pawlowna, deren diskontinuierliches Bewegungsmuster stellvertretend für viele andere diese Geschichte des 20. Jahrhunderts räumlich und dicht gedrängt vor Augen führt. Ihr Lebensweg wird als Bewegungsmuster in wenigen Worten nachgezeichnet:

Ich stieß auf Galina Pawlowna.

Eine Hymne auf die Füße von einst, bevor sie müd waren!

Nachdem sie hier angekommen war (in einem der Winter nach dem Krieg, Fritz wusste nicht mehr genau, in welchem), blieb sie auch gleich hängen, bis zum heutigen Tag, mehr als vierzig Jahre. Eine Russin, sagte sie vereinfachend. Sie kam aus der Ukraine, von den Deutschen nach Deutschland geraubt, danach von den Engländern beschlagnahmt und ums Haar zu Churchills Freund Stalin abgeschoben, sagte mir Fritz. Vorher noch von den Engländern bombardiert. In Amerika unerwünscht, nach Argentinien abgeschoben, von Buenos Aires in den Süden abgeschoben, in die Südspitze Patagoniens.

Verstehen Sie etwas von der Welt? ${ }^{19}$

Die Absurdität dieses gebrochenen, zerbrochenen Lebenslaufes, der freilich für Millionen von Menschen steht, ist Programm. Die sich an das knapp zusammengefasste Bewegungsmuster, das später durch die Darstellung der 'Behandlung' dieser Frau auf der Einwandererinsel Ellis Island vor New York ergänzt wird, anschließende Frage nach dem Verstehen, die sich nicht nur an den Protagonisten, sondern auch an das Lesepublikum richtet, führt die Bewegungen der Romanfiguren mit den vom Roman ausgelösten Verstehensprozessen explizit zusammen. Verstehen Sie etwas von dieser Welt?

Die absurde Diskontinuität eines Weges, der nie das Ergebnis frei getroffener Entscheidungen, sondern eines beständigen Gehandelt-Werdens, einer unablässigen Lebensbedrohung ist, stellt dem Leben überhaupt die Sinnfrage und verknüpft diese mit den Bewegungsfiguren des Romans. Denn hinter diesen Bewegungsfiguren wird die Hermeneutik brüchig. In der Tat sind die Romanfiguren vor allem dies: Figuren, die in erster Linie für eine je spezifische Art der Bewegung und des Verstehens einstehen. Der Erzählerkommentar selbst lässt hieran keinen Zweifel: „Jede Personenbeschreibung wäre ein Reisebericht.“20

19 Ebda., S. 42.

20 Ebda., S. 43. 
Der Reisebericht aber führt diese Versuche, einen Lebensweg zu verstehen, räumlich in seinen Bewegungen vor: Darin liegt seine Strahlkraft.

Auf dieser Ebene durchdringen sich die Reisen von anderen und das Schreiben über andere in einem Text, der deutliche reiseliterarische Züge besitzt. In den Reiseberichten der einzelnen Figuren erscheinen letztere als Figuren von Reisen, von denen der Roman nicht weniger als von der Reise des Ich-Erzählers berichtet. Denn erst die Kreisbewegung, die Rückkehr, ermöglicht das 'Mitbringen' all jener Geschichten, welche das Wenige bilden, das der Reisende den Daheimgebliebenen liefern kann. So ist Feuerland ein Reisebericht, hinter dessen Reise sich Berichte vieler Reisen und vieler weiterer Figuren auftun. Daraus ergibt sich die Gesamtstruktur des Textes. Denn das textgenerierende und bisweilen seriell und relational angewandte Prinzip ist die Reise, aus der viele andere Reisen hervorquellen, die ihrerseits andere Reisen verheißen. Ihr Ende muss abrupt sein, am Ende des Romans muss notwendig das Wörtchen „tot“ stehen, hörte die Bewegung doch sonst niemals auf und ginge ewig weiter. Was sie letzten Endes auch tut.

Das patagonische Land ist eine Einschreibefläche. Für den Protagonisten taugt Patagonien weder als Fluchtort noch als Gegen-Welt. Die 'wüste' Landschaft taugt nur als Fläche, auf der sich die Reisebewegungen der Romanfiguren einschreiben und auf der diese vom ersten Abschnitt des Romans an ihre „Lebenszeichen“ hinterlassen. Es ist freilich eine Fläche mit einer geschichtlichen Tiefe, einer Tiefe, die alte Vektoren, alte Bahnungen speichert. Die Fläche selbst aber ist in Stadlers Roman leer: Der in Feuerland aufgespannte literarische Raum erfasst nicht die Literatur, die sich in Argentinien selbst entwickelt hat; die expliziten intertextuellen Verweise beschränken sich auf die abendländische Literatur von der Bibel über Cervantes bis Chatwin, von Goethe über Stifter bis (intratextuell) Stadler selbst. Die Literaturen Lateinamerikas, die an den Literaturen des Abendlandes als Literaturen der Welt ${ }^{21}$ asymmetrisch partizipieren, bleiben hingegen vergessen und ausgespart, als hätte es am „Ende der Welt“ niemals Schriftstellerinnen und Schriftsteller gegeben: nur abendländische Reisende wie Chatwin, wie der Erzähler, die das verlorene Land in Erinnerung rufen. In diesem Sinne gilt: Patagonien ist auch für den Europäer Stadler in erster Linie eine Schreibfläche, welche eine (amerikanische) Leere repräsentiert, die von Europa her zu füllen ist und auch gefüllt wird.

Mag sein, dass aus diesem Grunde die Rückkehr des Ich-Erzählers nach Europa nicht den Abschluss des Romans bildet, sondern dass diese Rückkunft von

21 Vgl. hierzu Ette, Ottmar: WeltFraktale. Wege durch die Literaturen der Welt. Stuttgart: J.B. Metzler Verlag 2017. 
der 'Weltreise' vielfach in vorangehende Kapitel eingeblendet wird und gleichsam in den Text diffundiert. Die Schreibfläche bleibt von der ersten bis zur letzten Zeile Patagonien, dessen Name seit Antonio Pigafettas phantasievollem Reisebericht von Magalhães’ Weltumsegelung (1519-1522) für die Projektionen europäischer Träume nach Amerika einsteht. Doch die von dem italienischen Reisenden zu Anfang des 16. Jahrhunderts projizierten Riesen, die sogenannten Patagonier, auf die anlässlich des 'Tiermenschen' auch in Stadlers Roman angespielt wird, haben in Feuerland kleineren Menschen und bescheideneren Visionen Platz gemacht. Der reiseliterarische Ort des Abschieds, von dem aus noch eine letzte Hoffnung projiziert wird - „Lächerlich, ich weiß, aber so war es“²2 -, wird im bereits erwähnten vierunddreißigsten Kapitel gestaltet und betrifft geradezu selbstverständlich einen Ort interkontinentaler Verkehrsverbindungen, den internationalen Flughafen von Buenos Aires:

Auf dem Flughafen. Vor mir noch einmal eine Art Panorama. Ein Geruch wie über dem Hafen von Heraklion, dem Hafen, über den ja schon der Verkehr des Labyrinths von Knossos lief. Gerüche und Geräusche von Dingen, die hinter mir liegen. ${ }^{23}$

Bewusst werden hier die Strukturen mehrerer Häfen übereinander gelegt, die wie in einem Palimpsest nahezu gleichzeitig lesbar werden. Der Blick zurück, den der reiseliterarische Ort des Abschieds stets miteinschließt, verbindet sich mit einem Blick zurück auf die abendländische Antike, die Geschichte des Okzidents. Heraklion als Haupthafen Kretas verweist geschickt auf jenen anderen Ort der Insel, an dem einst Europa auf dem Rücken des Stieres nach der Überquerung des Meeres an Land gekommen war. Nicht als die strahlende Erscheinung, sondern als eine Migrantin, genauer eine Deportierte, eine vom Gotte Vergewaltigte, als die Ur-Mutter derer, die ihrer Heimat entrissen zu Heimatlosen, im nietzscheanischen Sinne folglich zu Europäern geworden sind.

Ausgehend von den Höhlen in der Nähe von Pico Grande assoziiert der IchErzähler auf Nachfragen seiner patagonischen Verwandten verschiedene Höhlen, die von der Bärenhöhle seiner süddeutschen Heimat bis zur „Diktehöhle [...], wo Zeus geboren war“, und zur „Idahöhle“ reichen, „wo er vor seinem Vater versteckt wurde, damit der ihn nicht gleich zu Beginn der Geschichte auffraß“. ${ }^{24}$ Von allem Anfang an sind die Geschichte und das Geschichtenerzählen im Abendland bedroht durch einen urplötzlichen Abbruch. Doch von diesem Ort gelangt der Erzähler unmittelbar zu „der Stelle, wo Europa an Land

22 Stadler, Arnold: Feuerland, S. 149.

23 Ebda.

24 Ebda., S. 45. 
getragen wurde“ und verweist darauf, dass „etwas südlicher“ - wohl auf der heute Malta genannten Insel - „Kalypso beheimatet gewesen“ und der Apostel „Paulus an Land gespült worden“ sei. ${ }^{25}$ Europa steckt voller Reisender, von der Odyssee bis zum Urchristentum: an Land gespült und wieder ausgespuckt.

Innerhalb der vom Text evozierten Abfolge von „Strandungsorten“26 kommt dem Verweis auf die Sage von Europa eine besondere Bedeutung zu. Denn Europa, die schöne Okeanide, nach der sich später ein Kontinent benannte, dessen räumliche Grenzen stets ungewiss blieben, war orientalischer Herkunft und zugleich Opfer einer Freiheitsberaubung in Tateinheit mit Vergewaltigung und Deportation. Europa war der Lust eines Gottes ausgesetzt, Opfer eines Augenblickes und bald vergessen. Von De-Portation und Deterritorialisierung sind nicht nur die verschiedenen Fassungen und Bearbeitungen der Sage Europas, sondern auch jene Geschichten geprägt, von denen der Ich-Erzähler uns aus Patagonien berichtet. Europa ist die Figur, die Modell steht für so viele fatale Migrationen einschließlich jener von Menschen des Südens, welche das Mittelmeer zu überqueren und nach der Welt im Norden gelangen wollen, die sie nicht kennen.

Doch zurück zur Familiengeschichte, die eine Menschheitsgeschichte ist. Die 'neue Heimat' in einem 'neuen Deutschland' weit südlich des Äquators hat die Träume der Auswanderer während der vergangenen hundert Jahre nicht erfüllt. Die Zerstörung des Mythos von Amerika als Fluchtpunkt und Zielort, diese Arbeit am Mythos, der sich auch die stets negativen Seitenblicke auf die Vereinigten Staaten zuordnen lassen, geht einher mit der Zerstörung Europas als vermeintlich unverbrüchlicher, Ursprung, Herkunft und Heimat bietender Einheit. Doch dieses Europa ist mit den eigenen Verwundungen beschäftigt.

Die Heimatlosigkeit wird zur eigentlichen Heimat und zur Grundlage aller Bewegungen der Figuren dieses Romans, die ausnahmslos scharf umrissene Bewegungsfiguren sind. Alle sind mit allen verbunden, eine Vorstellung, die der Erzähler obsessiv seiner Geliebten vorrechnet, da doch ,jeder von jedem abstammt, wie man vereinfachend sagt““ 27

Zwei Eltern, vier Großeltern, acht Urgroßeltern, 16 Ururgroßeltern, 32 Urururgroßeltern: im Jahr von Linas Geburt hattest du schon 256 Mütter und 256 Väter, immer gleich viel Mütter wie Väter. Noch eine Null dran, und du kannst sagen, daß du von allen abstammst, herrührst, weiterdämmerst: Väter-Mütter; Väter-Mütter ... ${ }^{28}$

25 Ebda.

26 Ebda.

27 Ebda., S. 129.

28 Ebda., S. 130. 
Die Familiengeschichte öffnet sich auf die Menschheitsgeschichte, wird zum Modell einer Welt, in welcher letztlich alle mit allen verwoben und verwandt sind. Eine solcherart skizzierte Genealogie läuft gerade nicht auf einen Stammbaum, auf eine Wurzel, auf eine gesicherte Herkunft hinaus, sondern auf die Verbindung aller mit allen. An die Stelle der ehemals vorherrschenden (Stamm-) Baumstruktur ist eine proliferierende, beschleunigt weiterwuchernde Netzstruktur und damit letztlich eine relationale Logik getreten, die andere Logiken - wie etwa die genealogisch-patriarchalische, sich aus dem Tiroler Einwanderer Schwanz herleitende Deszendenz - gewiss nicht ausschließt, aber relativiert und untereinander verbindet. Und über allen schwebt das verbindende Moment von Migration und Heimatlosigkeit.

Die unterschiedlichsten Räume vernetzen sich untereinander, ohne dies noch über Zentren bewerkstelligen zu müssen, die Kommunikation erfolgt von Raum zu Raum, zwischen Peripherie und Peripherie, zwischen Andenvorland und Alpenvorland. Feuerland ist weit entfernt und doch nah zugleich. In einer solchen Welt, die zugleich von höchster Beschleunigung und relationaler Bewegungslosigkeit geprägt wird, ist eine Unterscheidung zwischen dem Fremden und dem Eigenen nicht mehr möglich, macht keinen Sinn mehr. Differenzen bestehen fort, aber nicht mehr eine radikale Alterität. Keiner mehr ist des Anderen Anderer, der Fremde des Fremden. Denn nach dem sogenannten Jahrhundert der Migrationen, nach dem 20. Jahrhundert, eröffnet sich ein weiteres Jahrhundert, in welchem der homo migrans unser Zeitgenosse bleibt.

Arnold Stadler ist in einer ungeheuer kompakten Romanschöpfung eine Veranschaulichung jenes komplexen und in seinen Konsequenzen noch längst nicht ausgedachten Prozesses geglückt, wie die Neue Welt in der Alten als Neue erscheinen und in der Neuen zur Alten Welt werden konnte. Auf dieser Relationalität basiert sein Rückblick auf das 20. Jahrhundert und mehr noch auf den zentrierten Prozess der europäischen Moderne, mit dem dieser Kurzroman kurzen Prozess zu machen versucht, ohne dessen Kreisstruktur und Zirkelhaftigkeit doch ganz entgehen zu können.

In Feuerland ist das Abendland im Blick zurück nur aufgeschoben, aber nicht aufgehoben. Es bleibt eingebunden in eine Welt der Migrationen und produziert damit gleichsam notwendig jene Literaturen ohne festen Wohnsitz, welche zu einem festen Bestandteil der Literaturen der Welt geworden sind. Für den literarischen Reisebericht bedeutet dies, dass für ihn nicht nur wie in Jean Baudrillards Amérique die selbstgewollten und selbstgewählten Bewegungen an der Oberfläche zählen, sondern die geschichtliche Tiefendimension, die Last unserer Geschichte und Geschichten, noch immer präsent ist. Europa ist hinter dem Horizont nicht verschwunden. Indem das Leben vieler Menschen zu einem Reisebericht wird, verwandelt sich der Reisebericht in den privilegierten Schlüssel zur Lektüre von 
Leben, die sich der komplexen Überlagerung unterschiedlichster Bewegungen verdanken. Die Analyse von Reiseliteratur wird dann zum Modus der Erkenntnis einer Zeit, in welcher eine Poetik der Bewegung längst selbstverständlich geworden ist und alle Schichten unseres Verstehens durchdringt. Selbstverständlich ist sie nicht, weil sie keiner Begründung mehr bedürfte, sondern weil sie allgegenwärtig geworden ist in einer Epoche, in welcher sich nicht nur das Klima, die Biodiversität oder die Artenvielfalt wandeln, sondern auch - mit einem Wort Erich Auerbachs gesagt - die Orte des Menschen im Universum. 